\title{
A Position-Level Global Optimization Inverse Kinematic Solution Algorithm for Dual Redundant Robots Based on Motion Characteristics
}

\author{
Jingjie He iD \\ AICFVE of Beijing Film Academy, Beijing, China \\ Correspondence should be addressed to Jingjie He; hejingjie4@sina.com
}

Received 30 October 2017; Revised 22 January 2018; Accepted 14 February 2018; Published 26 March 2018

Academic Editor: Paula L. Zabala

Copyright (c) 2018 Jingjie He. This is an open access article distributed under the Creative Commons Attribution License, which permits unrestricted use, distribution, and reproduction in any medium, provided the original work is properly cited.

Camera robot is an important tool for realizing and reproducing complex camera motion path in modern special film effects. This paper proposed an inverse kinematics optimization algorithm for PRRPR-S redundant degrees of freedom (DoF) camera robot. This paper analyzed the motion characteristics, in Genetic Mix (GM) method, from the idea of movement boundary composed of part robot axis. Then proposed Simplify Mix (SM) method which can stably converge to the global optimal solution in a shorter time.

\section{Introduction}

1.1. Project Requirements Analysis. The PRRPR-S robot, referred to as camera robot, is an important tool of reproducing camera movement for multilayer composite film effects and realizing complex camera motion path. It is an important interactive node in film virtual manufacturing [1]. At present, the camera shooting trajectory is obtained by teaching mode, and the camera motion data in the preview system cannot be used directly. This paper studied the inverse kinematic algorithm of camera robot with global optimization ability, which makes camera move directly and transparently in a shorter time.

1.2. Theory Requirements Analysis. The algebraic equation form of redundancy serial robot inverse kinematics on velocity level is linear style [2], and the position-level is expressed as a nonlinear system and usually solved by numerical iteration. Liegeois [3] proposed gradient projection method based on generalized inverse matrix, which got the local optimal solution on velocity level but there was a cumulative error. Parker et al. [4] used genetic algorithm (GA) to solve the kinematic equations of redundant DoF manipulators. Sung et al. [5] took the terminal actuator positional deviation as the optimization target on the basis of the forward kinematics equation, which obtained the global optimal solution. The algorithm runs for a long time, and the multioptimization objective function is hard to construct.

Masayuki et al. [6] and Singh and Claassens [7] analyzed the single redundant DoF serial robot, which determined the self-motion space, and converted the redundancy into arm angle. The method is easy to construct the objective function.

The camera robot needs to get the position and attitude of the end-effector exactly in practice. And it has 8 joints, corresponding to high dimension of solution space, in other words, low effective solution proportion space. By using GA method based on forward kinematics equations, it is difficult to find valid solutions and attach other optimization goals. In addition, the randomness of the initial population makes the existence probability of the effective individuals very low, and the algorithm convergence effect is poor. Taking the axes of $r_{1}$ and $r_{4}$ as redundancy, Zhang et al. [8] proposed Genetic Mix (GM) method which include motion characteristics, GA based on redundant DoF and pattern search. By presetting the initial population, GM overcomes the defects of randomness and sequence in GA and converges to the global optimal solution stably on position-level. But in literature [8], there is no discussion about key analysis of how to get redundant DoF theoretical effect solution area. This paper, first, discussed the key analysis in detail, which 
TABLE 1: Robot link parameters.

\begin{tabular}{|c|c|c|c|c|}
\hline Transform & $\theta$ & $r$ & $l$ & $\alpha$ \\
\hline 0 & $\frac{\pi}{2}$ & 0 & 0 & $\pi$ \\
\hline & 2 & & & 2 \\
\hline 1 & 0 & $r_{1}+r_{10}$ & 0 & $-\frac{\pi}{2}$ \\
\hline 2 & $\theta_{2}-\frac{\pi}{2}$ & $r_{2}$ & 0 & $-\frac{\pi}{2}$ \\
\hline 3 & $\theta_{3}-\frac{\pi}{2}$ & 0 & $l_{3}$ & $-\frac{\pi}{2}$ \\
\hline 4 & 0 & $r_{4}+r_{40}$ & 0 & $\frac{\pi}{2}$ \\
\hline 5 & $\theta_{5}+\frac{\pi}{2}$ & 0 & 0 & $-\frac{\pi}{2}$ \\
\hline 6 & $\theta_{6}$ & $r_{6}$ & 0 & $\frac{\pi}{2}$ \\
\hline 7 & $\theta_{7}-\frac{\pi}{2}$ & 0 & 0 & $-\frac{\pi}{2}$ \\
\hline 8 & $\theta_{8}$ & 0 & 0 & 0 \\
\hline
\end{tabular}

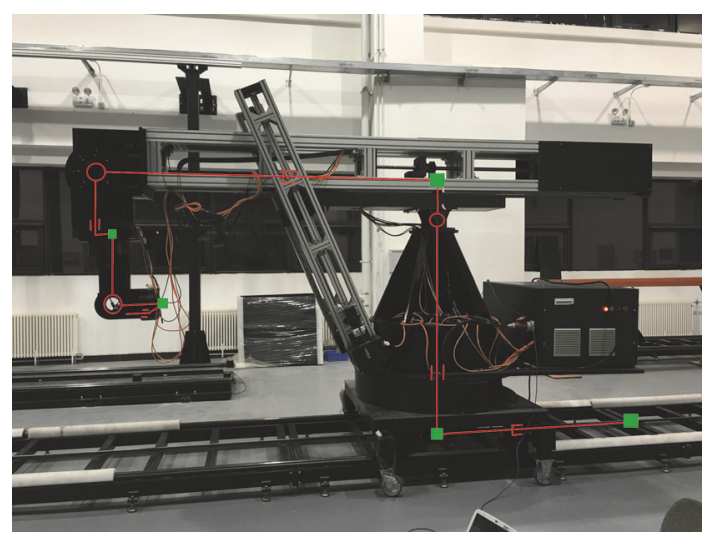

Figure 1: Camera robot.

regarded as the most extended/contract state, and, second, proposed Simplify Mix (SM) method which reduces the time consumption further.

\section{Motion Characteristic Analysis}

2.1. Camera Robot Workspace. The camera robot is shown in Figure 1 . There are 8 axes, including bottom linear axis $r_{1}$, ring style bottom rotate axis $\theta_{2}$, ring style top rotate axis $\theta_{3}$, top linear axis $r_{4}$, elbow axis $\theta_{5}$, rotate of wrist $\theta_{6}$, tilt of wrist $\theta_{7}$, and roll of wrist $\theta_{8}$. Modeled with $\mathrm{D}-\mathrm{H}$ method, the robot "initial standard zero state" and coordinate system on the link are shown in Figure 2.

The kinematic link parameters are shown in Table 1.

$r_{10}$ and $r_{40}$ are fixed value by mechanism. $S$ represents the shoulder point, $E$ represents the elbow point, $W$ represents the wrist point, and $U$ represents the upper arm point ( $U$ is not an axis).

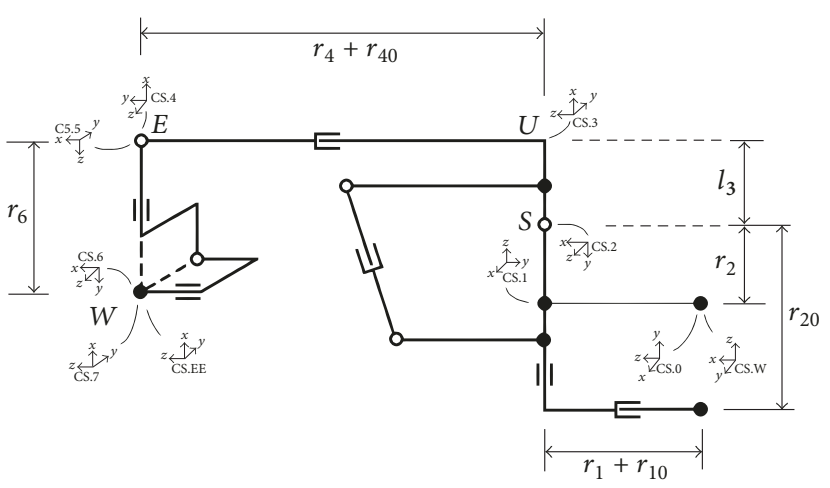

FIGURE 2: Schematic diagram of camera robot mechanism.

The DH matrices of camera robot are as follows:

$$
\begin{aligned}
& A_{0}=\left[\begin{array}{llll}
0 & 0 & 1 & 0 \\
1 & 0 & 0 & 0 \\
0 & 1 & 0 & 0 \\
0 & 0 & 0 & 1
\end{array}\right], \\
& A_{1}=\left[\begin{array}{cccc}
1 & 0 & 0 & 0 \\
0 & 0 & 1 & 0 \\
0 & -1 & 0 & r_{1}+r_{10} \\
0 & 0 & 0 & 1
\end{array}\right], \\
& A_{2}=\left[\begin{array}{ccccc}
\cos \left(\theta_{2}-\frac{\pi}{2}\right) & 0 & -\sin \left(\theta_{2}-\frac{\pi}{2}\right) & 0 \\
\sin \left(\theta_{2}-\frac{\pi}{2}\right) & 0 & \cos \left(\theta_{2}-\frac{\pi}{2}\right) & 0 \\
& 0 & -1 & 0 & r_{2} \\
0 & 0 & 0 & 1
\end{array}\right],
\end{aligned}
$$




$$
\begin{aligned}
& A_{3} \\
& =\left[\begin{array}{cccc}
\cos \left(\theta_{3}-\frac{\pi}{2}\right) & 0 & -\sin \left(\theta_{3}-\frac{\pi}{2}\right) & l_{3} \cdot \cos \left(\theta_{3}-\frac{\pi}{2}\right) \\
\sin \left(\theta_{3}-\frac{\pi}{2}\right) & 0 & \cos \left(\theta_{3}-\frac{\pi}{2}\right) & l_{3} \cdot \sin \left(\theta_{3}-\frac{\pi}{2}\right) \\
0 & -1 & 0 & 0 \\
0 & 0 & 0 & 1
\end{array}\right] \\
& A_{4}=\left[\begin{array}{cccc}
1 & 0 & 0 & 0 \\
0 & 0 & -1 & 0 \\
0 & 1 & 0 & r_{4}+r_{40} \\
0 & 0 & 0 & 1
\end{array}\right] \text {, } \\
& A_{5}=\left[\begin{array}{cccc}
\cos \left(\theta_{5}+\frac{\pi}{2}\right) & 0 & -\sin \left(\theta_{5}+\frac{\pi}{2}\right) & 0 \\
\sin \left(\theta_{5}+\frac{\pi}{2}\right) & 0 & \cos \left(\theta_{5}+\frac{\pi}{2}\right) & 0 \\
0 & -1 & 0 & 0 \\
0 & 0 & 0 & 1
\end{array}\right] \text {, } \\
& A_{6}=\left[\begin{array}{cccc}
\cos \left(\theta_{6}\right) & 0 & \sin \left(\theta_{6}\right) & 0 \\
\sin \left(\theta_{6}\right) & 0 & -\cos \left(\theta_{6}\right) & 0 \\
0 & 1 & 0 & r_{6} \\
0 & 0 & 0 & 1
\end{array}\right] \text {, } \\
& A_{7}=\left[\begin{array}{cccc}
\cos \left(\theta_{7}-\frac{\pi}{2}\right) & 0 & -\sin \left(\theta_{7}-\frac{\pi}{2}\right) & 0 \\
\sin \left(\theta_{7}-\frac{\pi}{2}\right) & 0 & \cos \left(\theta_{7}-\frac{\pi}{2}\right) & 0 \\
0 & -1 & 0 & 0 \\
0 & 0 & 0 & 1
\end{array}\right] \text {, } \\
& A_{8}=\left[\begin{array}{cccc}
\cos \left(\theta_{8}\right) & \sin \left(\theta_{8}\right) & 0 & 0 \\
-\sin \left(\theta_{8}\right) & \cos \left(\theta_{8}\right) & 0 & 0 \\
0 & 0 & 1 & 0 \\
0 & 0 & 0 & 1
\end{array}\right] \text {. }
\end{aligned}
$$

The values of Table 1 are as follows: $r_{10}=1000, r_{2}=300$, $l_{3}=333, r_{40}=2500$, and $r_{6}=963$. Then giving axes value, any posture of robot link can be calculated by transfer equations. For example, the posture of end-effector is

$$
T_{e}=A_{0} \cdot A_{1} \cdot A_{2} \cdot A_{3} \cdot A_{4} \cdot A_{5} \cdot A_{6} \cdot A_{7} \cdot A_{8} .
$$

Temporarily assume that the end-effector of the camera robot can get any posture through the wrist. As the robot moving in the bottom linear rail, the reachable space of $W$ in the plane perpendicular to the rail represents the robot workspace. Based on the transfer matrix, as (2), the workspace coordinates of the key points and boundary equation can be calculated, as shown in Figure 3. The red curve is the outer boundary, and the green curve is the inner boundary.

2.2. Redundancy Setting. Considering that the industrial 6DoF robot has mature algorithm of inverse kinematic solution, the bottom linear axis $\left(r_{1}\right)$ and the top linear axis $\left(r_{4}\right)$ are chosen as redundancy. After arbitrarily determining a set of

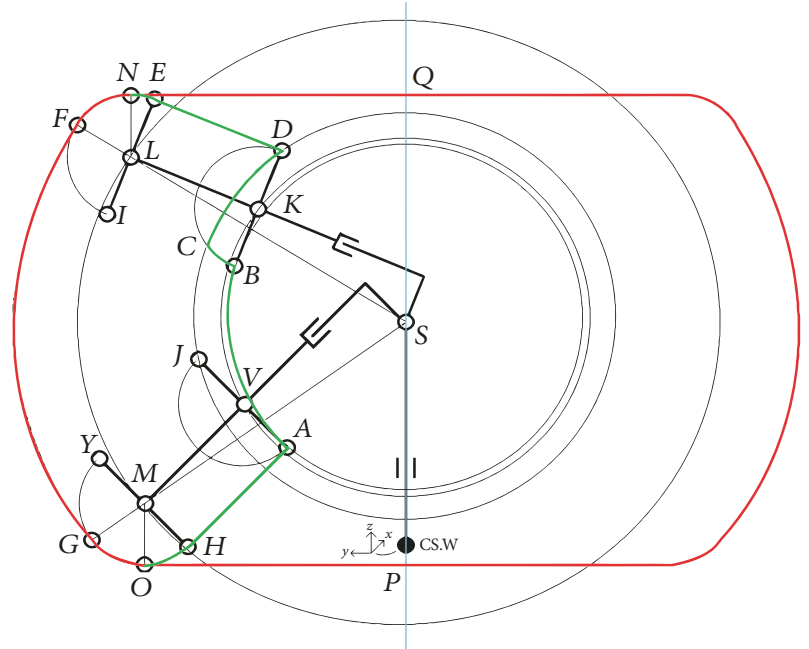

FIGURE 3: Workspace boundary of the camera robot.

$\left[\begin{array}{ll}r_{1} & r_{4}\end{array}\right]$, countable inverse solutions can be obtained. Choose the solution with the smallest value of the optimal function as the optimal solution, which reduces the 8-dimensional solution space to 2-dimensional one.

For clarity, declare the axis physical motion range as physical constraint and geometric constraint as theoretical constraint. The intersection of two constraints is the range which contains effective solution, called comprehensive constraint.

When using GA method with physical constraints of $\left[\begin{array}{ll}r_{1} & r_{4}\end{array}\right]$ for the gene range, due to the fact that the wrist attitude adjustment mechanism may block the view of camera, the effective solution share is very low and the algorithm is inefficient. In order to solve this problem, it is necessary to further analyze dual redundancy effective range. The basic idea is that, after a given posture, the robot should have two ways as "most extended state" and "the most contract state" to achieve the specified posture.

2.3. Analysis of $r_{1}$ Comprehensive Constraint. Note the following:

(1) WS is the distance between shoulder point $(S)$ and wrist point $(W)$.

(2) $p T$ is a 3-dimensional vector and represents the coordinate of target point position.

(3) $t S$ is the trace of shoulder point.

As for the rail can be extended to any length, the physical constraint of $r_{1}$ is not in consideration.

At different heights, there are specific theoretical maximum $W S, T W S_{\max }$, and the theoretical minimum $W S$, $T W S_{\min }$, as shown in Figure 4.

2.3.1. $W S_{\text {min }}$ Determination. Ask $p T$ perpendicular to $t S$, as dpTtSM, and the foot is ppTtSM. If $d p T t S M \leq T W S_{\min }$, refer to the lateral deviation of $p T$ that is not big enough, as $T_{2}$ shown in Figure 5. There is $W S_{\min }=T W S_{\min }$. 


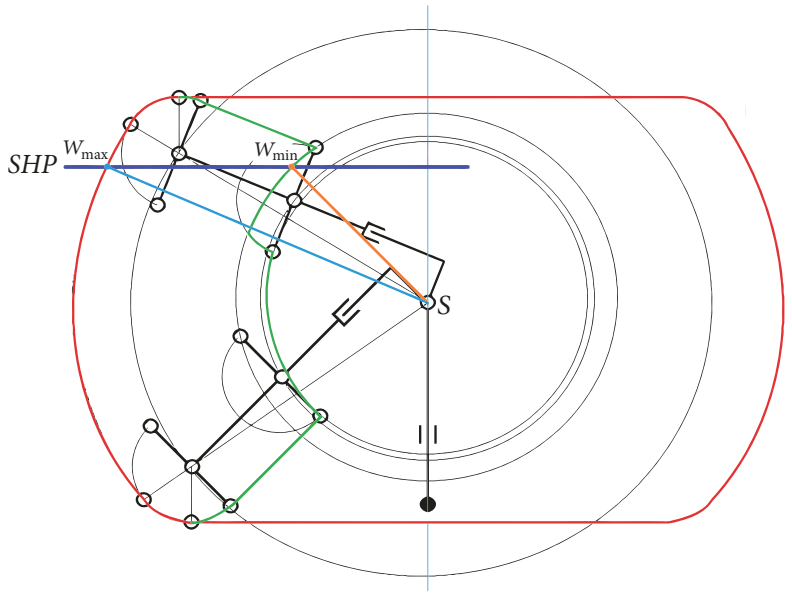

FIGURE 4: Theoretical $\max / \min W S$.

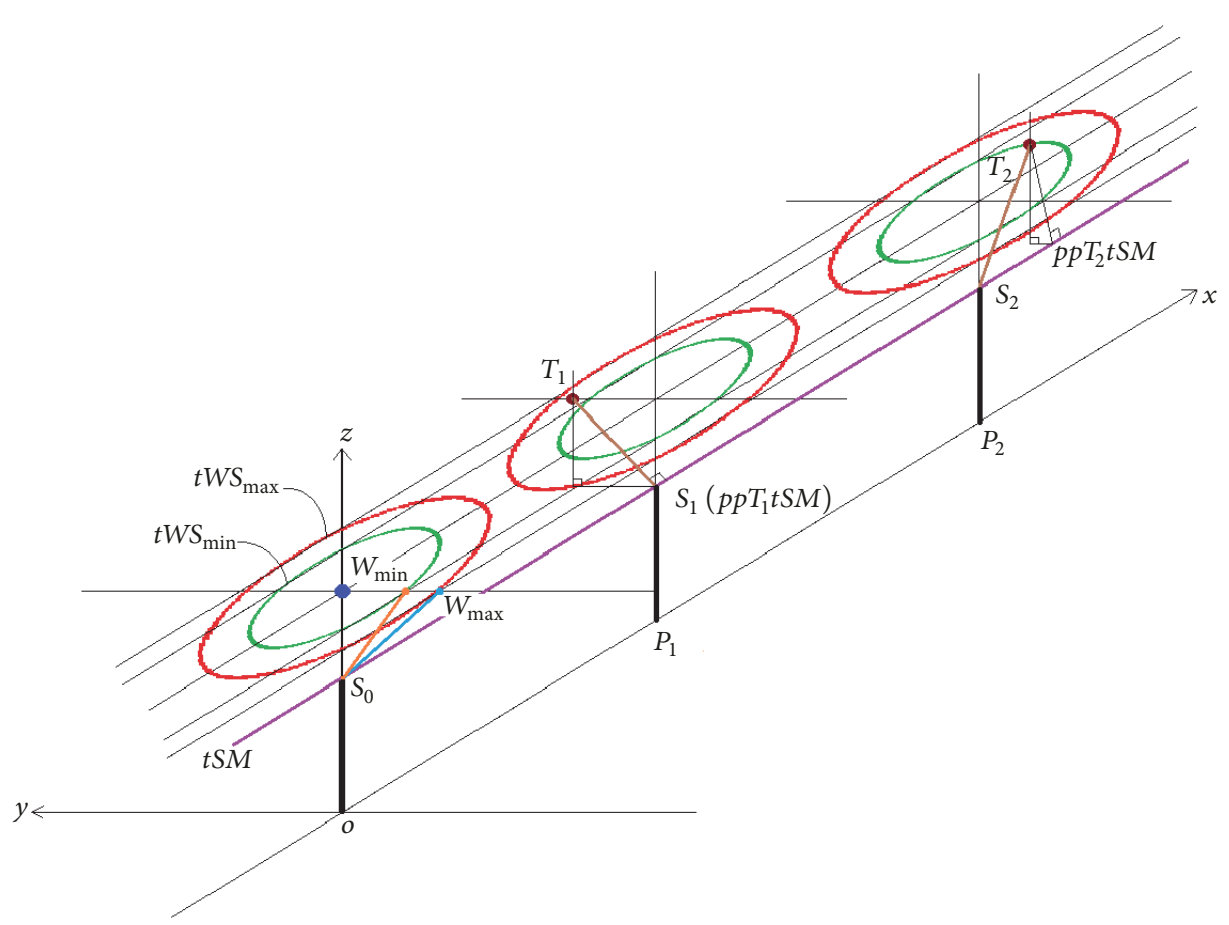

FIGURE 5: Reaching different $p T$ at the specified height.

If $T W S_{\min }<d p T t S M \leq T W S_{\max }$, refer to the lateral deviation of target point that is big enough, as $T_{1}$ shown in Figure 5. There is $W S_{\min }=\left\|\mathbf{p} \mathbf{T}-\mathbf{S}_{\mathbf{1}}\right\|$.

Thus, $W S_{\min }$ can be determined based on the $p T$.

When the camera robot reaches $p T$ in the most contract state, there are three cases according to the height of the target point and the degree of lateral deviation, as shown in Figure 6. According to $d p T t S M$ and $W S_{\text {min }}$, the limit position of $S$ can be obtained, and then determine the limit value of $r_{1}$.

2.3.2. WS $S_{\max }$ Determination. When the camera robot reaches $p T$ in the most extended state, $W$ must be on the arc, in which
$T W S_{\max }$ is the radius and the projection of $S$ on horizon plane passed $p T$ is the center, as shown in Figure 7.

According to $d p T t S M$ and $T W S_{\max }$, obtain the limit position of $S$, so as to determine the limit value of $r_{1}$. In summary, according to $p T$ as input, $r_{1}$ range is

$$
r_{1} \in\left[r_{1 \text { farn }}, r_{1 \text { closen }}\right] \cup\left[r_{1 \text { closep }}, r_{1 \text { farp }}\right] \text {. }
$$

\subsection{Analysis of $r_{4}$ Comprehensive Constraint}

2.4.1. The Factors of $r_{4}$ Determination Analysis. After arbitrarily determining $r_{1}$ in the comprehensive constraint, there are four factors that limit the value of $r_{4}$ : 


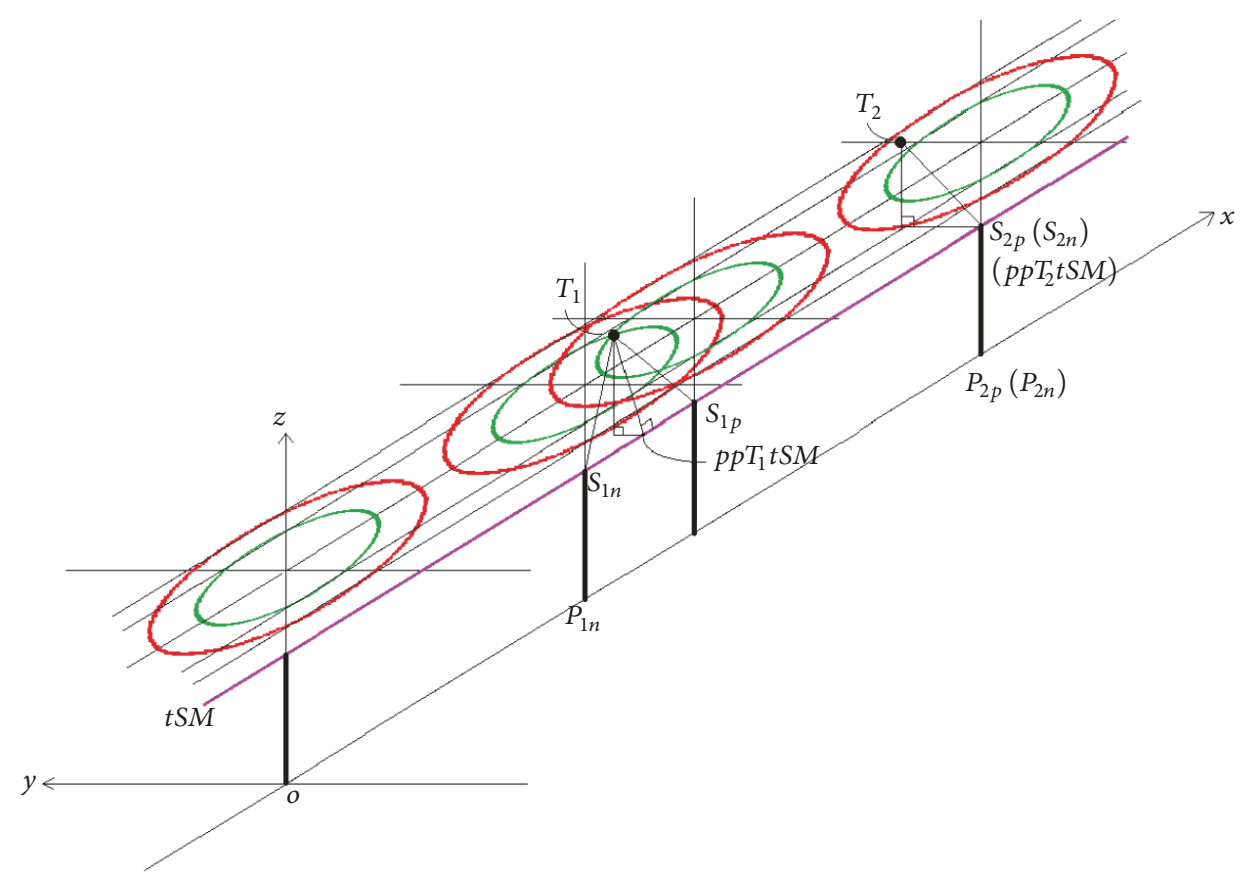

Figure 6: Reaching $p T$ with $W S_{\min }$.

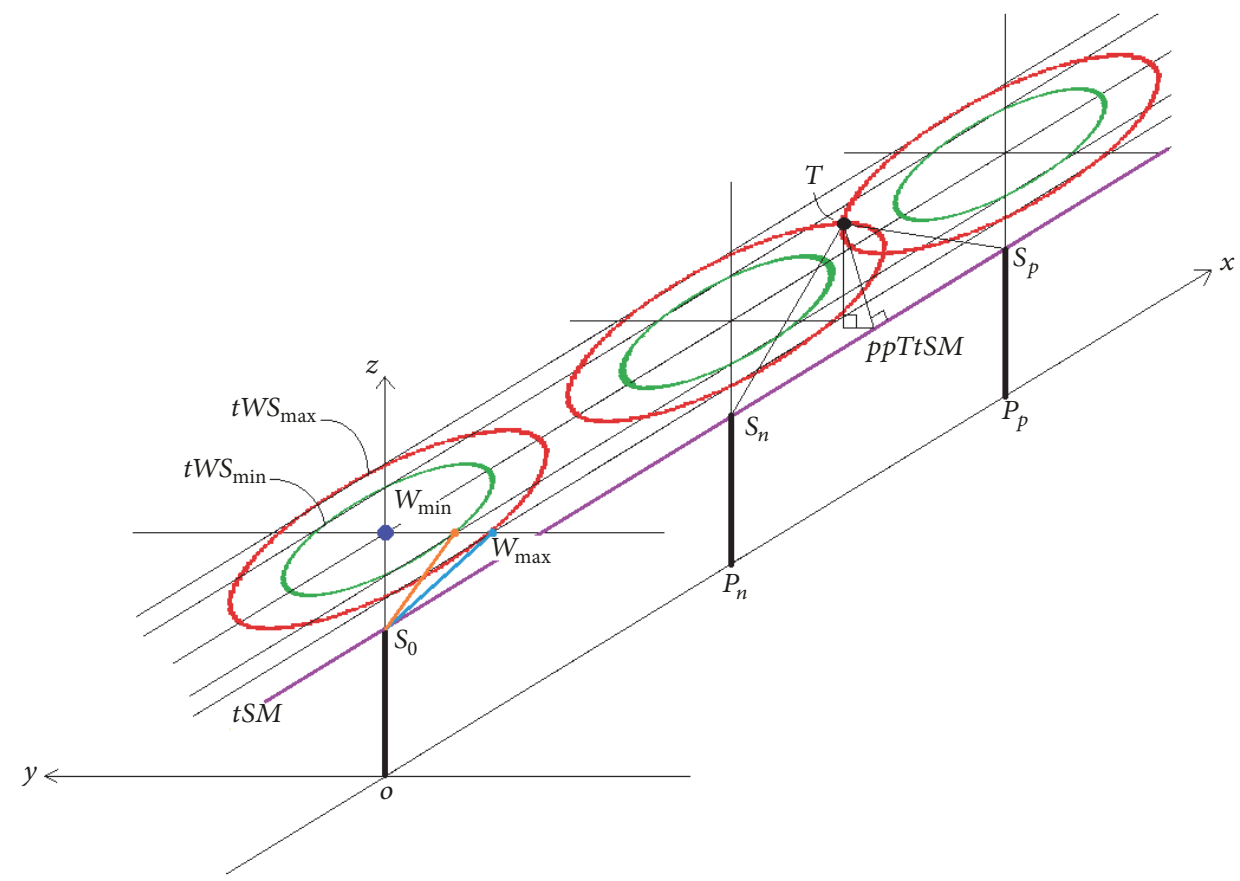

Figure 7: Reaching $p T$ with $W S_{\min }$.

(1) $S E, W E$, WS constitute a generalized triangle, as $r_{4} \in$ $\left[r_{4 \operatorname{minsf} 1}, r_{4 \operatorname{maxsf} 1}\right]$.

(2) UE moves within its physical constraint, as $r_{4} \in$ $\left[r_{4 \min }, r_{4 \max }\right]$.

(3) $\theta_{5}$ moves within its physical constraint, as $r_{4} \in$ $\left[r_{4 \text { minsf } 3}, r_{4 \text { maxsf } 3}\right]$.
(4) $\theta_{3}$ moves within its comprehensive constraint, as $r_{4} \epsilon$ $\left[r_{4 \operatorname{minsf} 4}, r_{4 \operatorname{maxsf} 4}\right]$.

The inclusion relation of factor sets is

$$
\begin{aligned}
{\left[r_{4 \operatorname{minsf} 4}, r_{4 \operatorname{maxs} 4}\right] } & \subseteq\left[r_{4 \operatorname{minsf3} 3}, r_{4 \operatorname{maxf} 3}\right] \\
& \subset\left[r_{4 \operatorname{minsf} 1}, r_{4 \operatorname{maxsf} 1}\right] .
\end{aligned}
$$




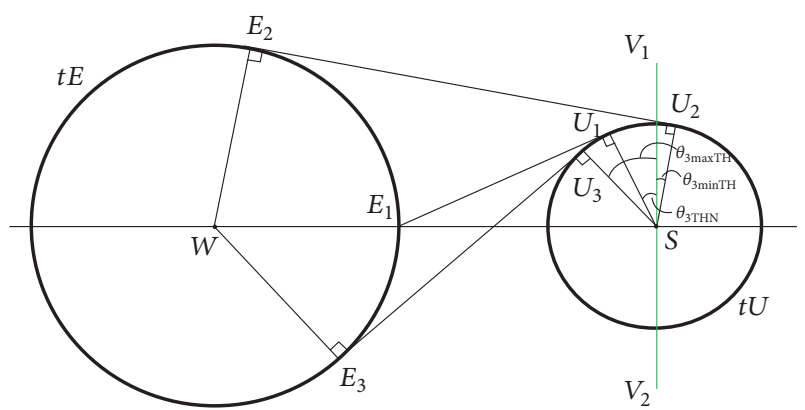

FIgURE 8: The physical limit state of $\theta_{3}$ when WS is horizontal.

That factor 4 included in factor 3 will be approved in the following context. Thus while $p T$ is in the workspace and $S$ is determined, there are

$$
r_{4} \in\left[r_{4 \min }, r_{4 \max }\right] \cap\left[r_{4 \operatorname{minsf} 4}, r_{4 \operatorname{maxsf} 4}\right]
$$

Select an arbitrary $r_{4}$ in this range which can guarantee that at least one effective inverse solution is obtained. So it is important to analyze impact of factor 4 to $r_{4}$.

2.4.2. Analysis of Factor 4. Given the target posture, $S$ is fixed after selecting $r_{1}$. For $W E$ is fixed, $E$ must be on the circle $t E$, whose center is $W$ and radius is WE. Similarly, US length is fixed, so $U$ point must be on the circle $t U$. $\angle E U S$ is right angle as mechanism set. $U E$ length is $r_{4}+r_{40}$, as shown in Figure 8. Regardless of the height of $W, W S$ length is the main factor affecting the length of the $U E$.

Take WS in horizontal as the benchmark situation. When $\theta_{3}$ rotates and $E$ is on the connection of $W S, U E$ gets smallest length on the WS connection. When WE is perpendicular to $U E, U E$ takes the extreme value. Since $\theta_{5}$ physical constraint is $[-\pi, 0]$, the limits of $r_{4}$ by $\theta_{3}$ theoretical constraint at WS in horizontal state and by $\theta_{5}$ physical constraint are the same.

According to the geometric principal, $\theta_{3}$ theoretical constraint is determined:

$$
\begin{aligned}
\theta_{3 \operatorname{minTH}}= & \frac{\pi}{2}-\arctan \left(\frac{\sqrt{W S^{2}-\left(r_{6}-l_{3}\right)^{2}}}{l_{3}}\right) \\
& -\arccos \left(\frac{W S^{2}-r_{6}^{2}+r_{6} \cdot l_{3}}{\sqrt{W S^{2}-r_{6}^{2}+2 \cdot r_{6} \cdot l_{3}} \cdot W S}\right), \\
\theta_{3 \operatorname{maxTH}}= & \pi-\left[\left(\frac{\pi}{2}-\angle E_{3} S W\right)+\angle E_{3} S U_{3}\right] \\
= & \frac{\pi}{2}+\angle E_{3} S W-\angle E_{3} S U_{3} \\
= & \frac{\pi}{2}
\end{aligned}
$$

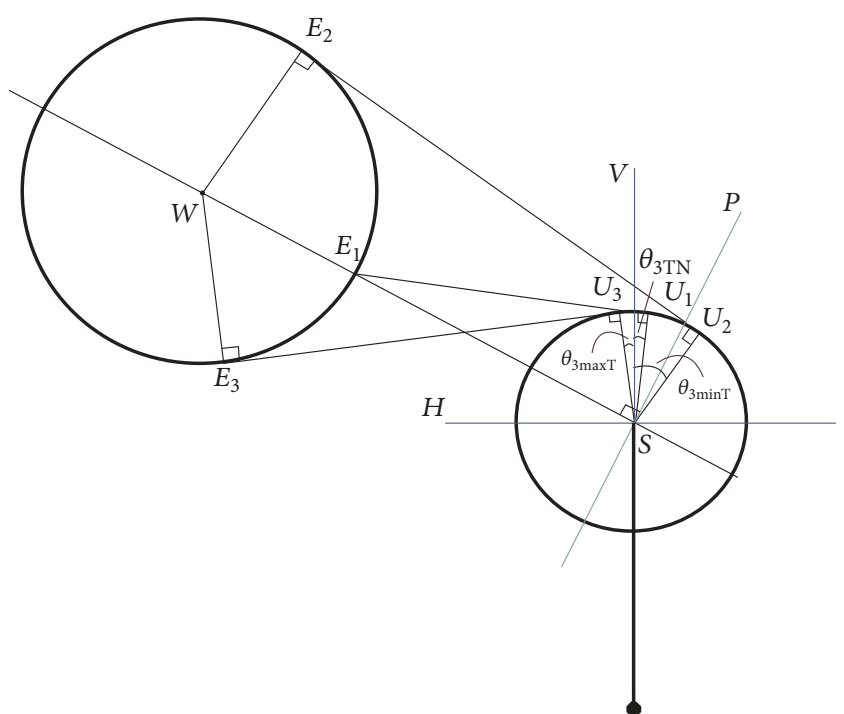

FIGURE 9: WS in general case.

$$
\begin{aligned}
& +\arccos \left(\frac{W S^{2}-r_{6}^{2}-r_{6} \cdot l_{3}}{\sqrt{W S^{2}-r_{6}^{2}-2 \cdot r_{6} \cdot l_{3}} \cdot W S}\right) \\
& -\arctan \left(\frac{\sqrt{W S^{2}-\left(r_{6}+l_{3}\right)^{2}}}{l_{3}}\right) .
\end{aligned}
$$

The general case when WS at an arbitrary angle to the horizontal plane is shown in Figure 9.

$P S$ is perpendicular to $W S$. While specifying $p T$, the angle of WS and the horizontal plane $H$ is $\angle W S H$. There are

$$
\begin{gathered}
\theta_{3 \operatorname{maxT}}=\theta_{3 \operatorname{maxTH}}+\angle W S H, \\
\theta_{3 \operatorname{minT}}=\theta_{3 \operatorname{minTH}}+\angle W S H, \\
\theta_{3 \mathrm{TN}}=\theta_{3 \mathrm{THN}}+\angle W S H .
\end{gathered}
$$

In general, when specifying $p T$ and $S$, three theoretical characteristic positions of $\theta_{3}$ are obtained. Since $\theta_{3}$ has a physical constraint, as $\theta_{3 \min } \leq \theta_{3} \leq \theta_{3 \max }$. Therefore, we need to take $\theta_{3}$ comprehensive constraint intervals and position of $\theta_{3 \mathrm{THN}}$ into account.

2.4.3. TRFour Function. Before interpartition analysis, discuss the function:

$$
r_{4 \mathrm{~T}}=\operatorname{TRFour}\left(W, S, \theta_{3}\right) .
$$

This function may get $r_{4}$ that is outside the physical constraint. First, obtain $\theta_{3 \mathrm{TH}}$ corresponding to the WS horizontal state:

$$
\theta_{3 \mathrm{TH}}=\theta_{3}-\angle W S H \text {. }
$$

Then,

$$
\angle W S U=\left|\frac{\pi}{2}-\theta_{3 \mathrm{TH}}\right|
$$


In $\triangle W S U$, by cosine theorem, there is

$$
W U=\sqrt{W S^{2}+U S^{2}-2 \cdot W S \cdot U S \cdot \cos (\angle W S U)} .
$$

At the same time, there is

$$
\begin{aligned}
& \angle W U S=\arccos \left(\frac{W U^{2}+U S^{2}-W S^{2}}{2 \cdot W U \cdot U S}\right), \\
& \angle W U E=\left|\angle W U S-\frac{\pi}{2}\right| .
\end{aligned}
$$

In $\triangle W U E$, by cosine theorem, there is

$$
W E^{2}=W U^{2}+U E^{2}-2 \cdot W U \cdot U E \cdot \cos (\angle W U E) .
$$

Considering position of line with $U E$, there is

$$
\begin{aligned}
U E= & W U \cdot \cos (\angle W U E) \\
& -\sqrt{W E^{2}-W U^{2} \cdot \sin (\angle W U E)^{2}}=r_{4 \mathrm{~T}}+r_{40} .
\end{aligned}
$$

The result is

$$
r_{4 \mathrm{~T}}=U E-r_{40}
$$

2.4.4. Analysis of $\theta_{3}$ Effective Comprehensive Constraint. After determining $W$ and $S$, discuss $\theta_{3}$ comprehensive constraint. Based on this, discuss $r_{4}$ comprehensive constraint.

The blue sector (filled with upper right oblique line) is $\theta_{3}$ theoretical constraint. The red sector (filled with upper left oblique line) is $\theta_{3}$ physical constraint. The green sector (filled with cross line) is the overlapping area, which refers to comprehensive constraint. The purple line is $\theta_{3 \mathrm{TN}}$ position. Among them, the limit position and scale of $\theta_{3}$ physical constraint do not change, and there always is $\theta_{3 \operatorname{minT}}<\theta_{3 \mathrm{TN}}<$ $\theta_{3 \max T}$.

According to the following principles, get $r_{4 \operatorname{maxsf} 4}$ and $r_{4 \text { minsf } 4}$ corresponding to the value of $\theta_{3}$ :

(A) Effective $\theta_{3}$ exists in the intersection (green sector).

(B) With $\theta_{3 \mathrm{TN}}$ as the boundary, the theoretical constraints on both sides are monotonically increasing space.

As the standard examples, Figures 10(b) and 10(c) will be described. Figures $10(a)$, and $10(d)-10(j)$ can be treated as the same way as examples.

When $\theta_{3 \max }>\theta_{3 \max T}, \theta_{3 \max T} \geq \theta_{3 \min }>\theta_{3 \min \mathrm{T}}$ and $\theta_{3 \operatorname{minT}}<\theta_{3 \mathrm{TN}} \leq \theta_{3 \mathrm{~min}}$. There is an intersection of the lower subinterval of $\theta_{3}$ physical constraint and the higher subinterval of $\theta_{3}$ theoretical constraint, as shown in Figure 10(b). There are

$$
\begin{aligned}
& \theta_{3 \operatorname{minTP}}=\theta_{3 \min }, \\
& \theta_{3 \operatorname{maxTP}}=\theta_{3 \max \mathrm{T}} .
\end{aligned}
$$

And when $\theta_{3}=\theta_{3 \operatorname{minTP}}$, obtain $r_{4 \text { minsf } 4}$.

When $\theta_{3}=\theta_{3 \operatorname{maxT}}$, obtain $r_{4 \operatorname{maxsf} 4}$.

When $\theta_{3}>\theta_{3 \max T}, \theta_{3 \max T} \geq \theta_{3 \min }>\theta_{3 \operatorname{minT}}$ and $\theta_{3 \min }<\theta_{3 \mathrm{TN}}<\theta_{3 \operatorname{maxT}}$. There is an intersection of the lower subinterval of $\theta_{3}$ physical constraint and the higher subinterval of $\theta_{3}$ theoretical constraint, as shown in Figure 10(c). There are

$$
\begin{aligned}
& \theta_{3 \operatorname{minTP}}=\theta_{3 \min }, \\
& \theta_{3 \operatorname{maxTP}}=\theta_{3 \operatorname{maxT}} .
\end{aligned}
$$

And when $\theta_{3}=\theta_{3 \mathrm{TN}}$, obtain $r_{4 \text { minsf } 4}$.

There is

$$
\begin{aligned}
& r_{4 \operatorname{maxsf} 4}=\max \left(\operatorname{TRFour}\left(W, S, \theta_{3 \operatorname{minTP}}\right),\right. \\
& \left.\operatorname{TRFour}\left(W, S, \theta_{3 \operatorname{maxTP}}\right)\right) .
\end{aligned}
$$

In summary, on the basis of the boundary of the camera robot workspace, $r_{1}$ comprehensive constraint is determined according to $p T$ with $W S$ characteristic as benchmark. After selecting $r_{1}$, the influencing factors of $r_{4}$ are analyzed synthetically, and $r_{4}$ comprehensive constraint is obtained. And this is also the conception of sequence of the two redundancy in GA.

\section{Optimized Method SM}

3.1. Optimized Objective Function. The optimization goal is to improve the motion stability of the end-effector; this paper sets the motor shaft to move as little as possible with the higher load inertia. The optimization objective function $F$ is

$$
F=\mathbf{W} \cdot \mathbf{X}
$$

where $\mathbf{W}$ is the weight vector and $\mathbf{X}$ represents the movement distance vector of axis.

3.2. Algorithm Optimization. According to the camera robot motion characteristics, $r_{1}$ can be determined first and then the theoritical effect range of $r_{4}$ can be calculated. This is the sequence of two variables. GM method used the dual redundancy as genes. By setting the initial population with average distribution in effect solution area, GM can eliminate the large iterative calculation from GA nonlinear constraints caused by sequence of dual redundancy and overcome the low effective proportion of stochastic initial population. Then through the pattern search algorithm, the composite algorithm can converge to the global optimal solution stably.

In the experiment, it is found that the optimal solution of GM did not change or change little in the process of iterative computation. That is, the analysis of camera robot motion characteristics with average initial population distribution greatly weakened the global optimization effect of GA in GM. So it is desirable to solve in a shorter time by motion characteristics with pattern search. This paper proposed SM method. To verify the effect of it, set the experiment.

The robot is in the initial standard zero state. 4 to 8 meters in the positive direction of the rail, set 45 target postures evenly. Specifically, five planes are set in the space, and 9 target postures are distributed in each plane, as shown in Figure 11, where red line represents the coordinate system $z$-axis and black line represents the coordinate system $x$-axis. 


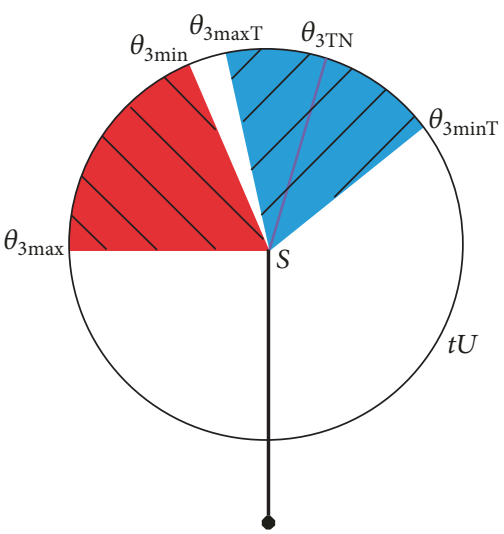

(a) Relationship-1

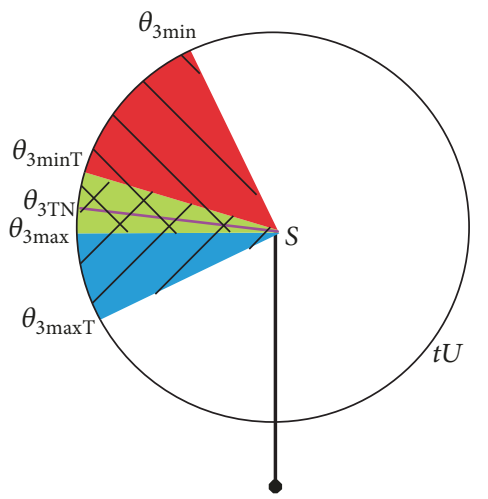

(d) Relationship-4

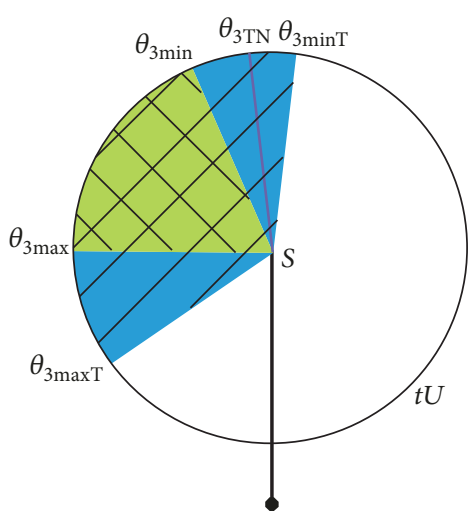

(g) Relationship-7

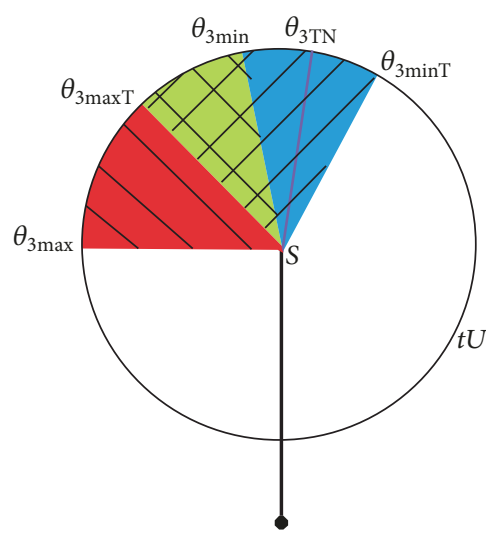

(b) Relationship-2

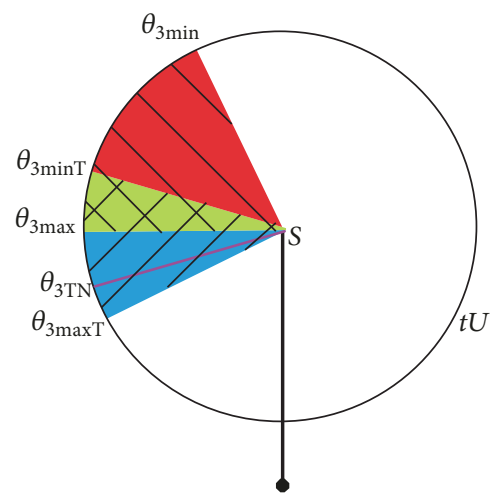

(e) Relationship-5

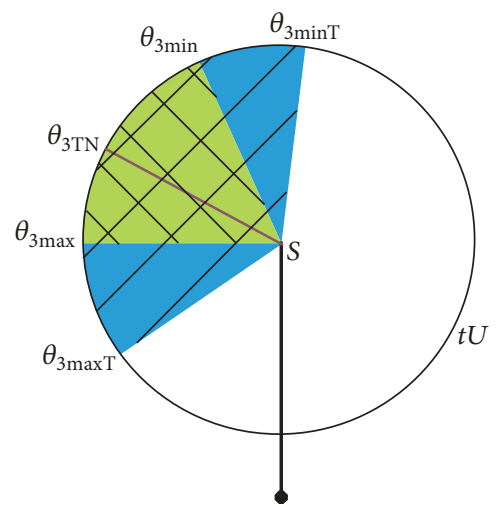

(h) Relationship-8

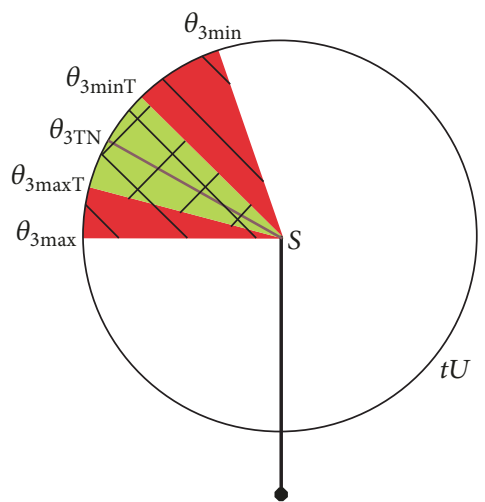

(j) Relationship-10

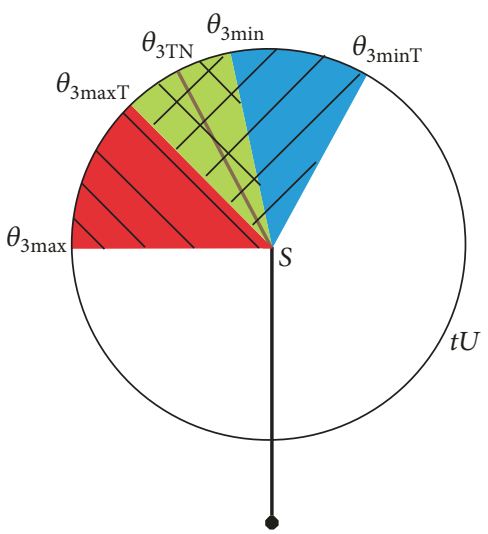

(c) Relationship-3

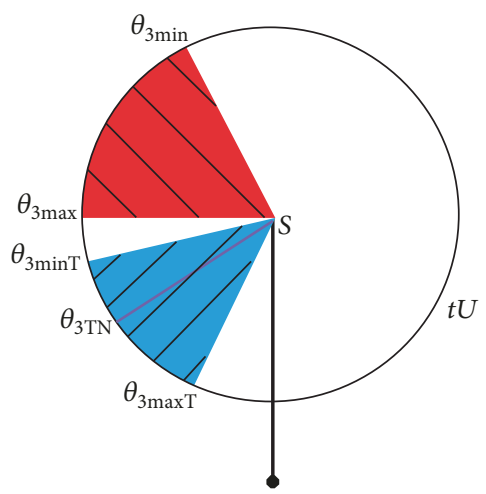

(f) Relationship-6

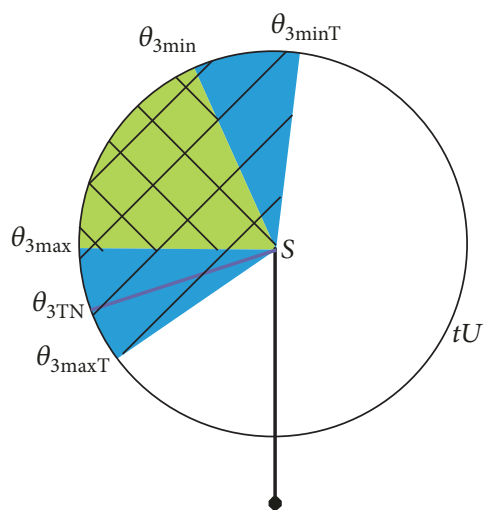

(i) Relationship-9

FIGURE 10: $\theta_{3}$ comprehensive constraint and $\theta_{3 \mathrm{THN}}$ relationship. 


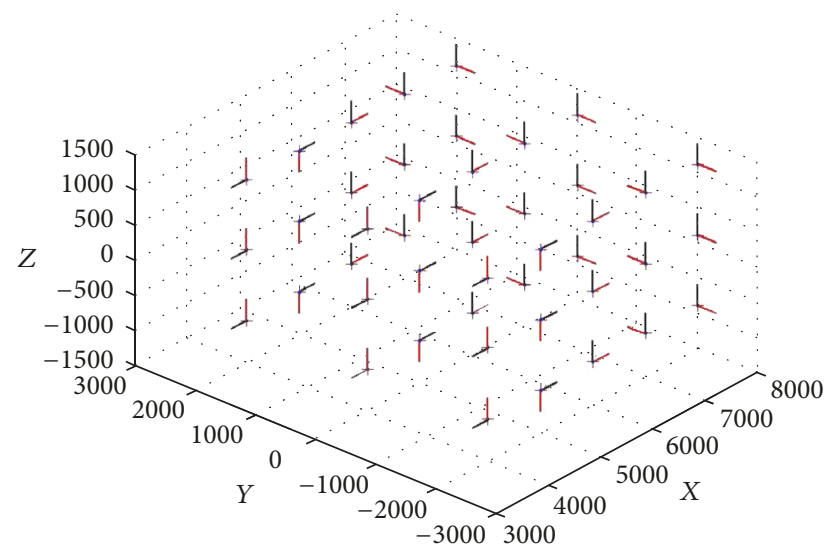

FIGURE 11: Target postures in workspace.

Assume that both $v s G M$ and $v s S M$ are 8-dimensional vectors and are solutions for each method, which can both achieve the target posture.

Set the physical constraints of each axis as

$$
\mathbf{v A R}=\left[\begin{array}{llll}
v A R_{1} & v A R_{2} & \cdots & v A R_{8}
\end{array}\right],
$$

where

$$
\begin{aligned}
v A R_{i}= & x_{j \max }-x_{j \min }, \\
& i=1,2, \ldots, 8, x_{j}=r_{1}, \theta_{2}, \theta_{3}, r_{4}, \theta_{5}, \theta_{6}, \theta_{7}, \theta_{\mathrm{EE}} .
\end{aligned}
$$

Define

$$
n D R=\prod_{i=1}^{8}\left[\frac{\left|v s G M_{i}-v s S M_{i}\right|}{v A R_{i}}\right] .
$$

$n D R$ can be understood as the proportion of the generalized distance between different solutions in the entire workspace.

Take the experiment as follows.

Select 20 values in $r_{1}$ comprehensive effective subinterval (specified $p T$ as boundary, the robot at the same side with the current state). According to $20 r_{1}$ values, the corresponding $r_{4}$ comprehensive constraints are calculated. Select the middle value as $r_{4}$ value, forming an individual with the corresponding $r_{1}$.

Calculate the optimal solutions by GM and SM methods, respectively. The result is shown in Figure 12. The $x$-axis is the target posture number, and $y$-axis is $n D R$. The solid point on the corresponding coordinate of the $x$-axis indicates that the GM gets a better solution, and the triangle represents that the SM gets a better solution. The cross symbol means that the two methods get the same solution.

For 45 target postures, GM costs $6328.32 \mathrm{~s}$ and SM costs $3410.07 \mathrm{~s}$. There is only one posture with big distance appearing on the 30th posture, where the ratio of two values of optimal objective function is $12 \%$, and GM method gets a better solution. The robot states are shown in Figure 13, the black body is the result of the GA, and the blue body is the result of the SM. The actual difference of two solutions is

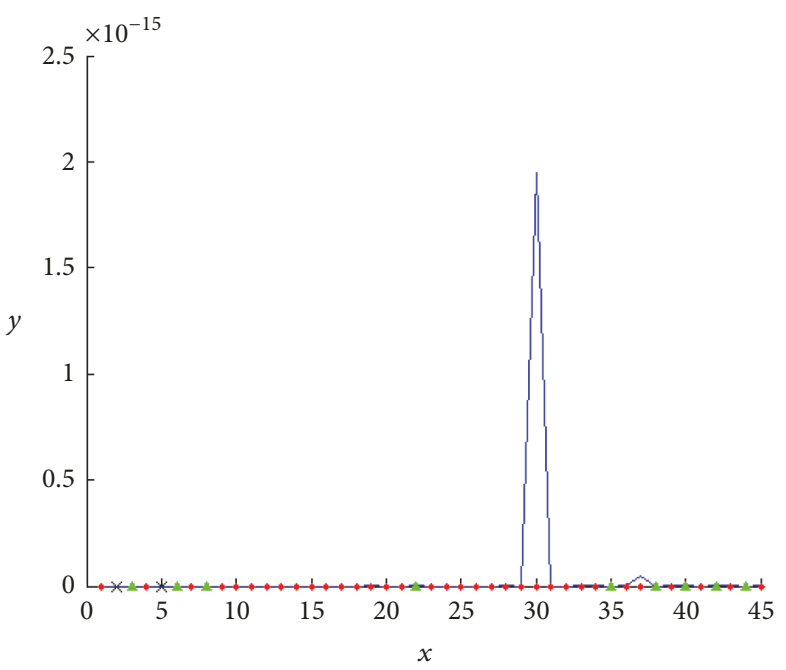

FIGURE 12: Compared result of two methods.

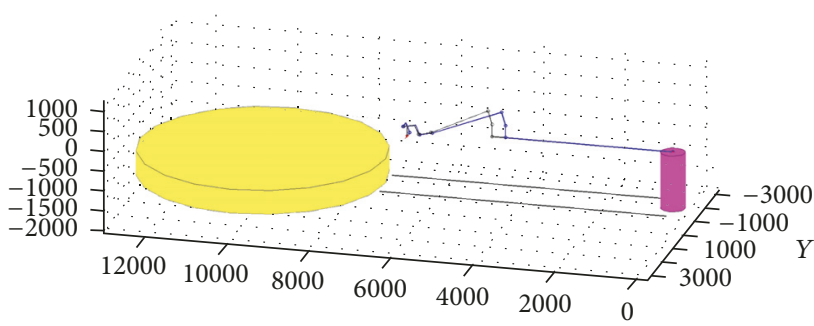

FIGURE 13: Maximum deviation of two methods.

not big. The solutions of two methods for the other 44 target postures are close enough to the global optimal solution (verified by a certain accuracy of the traversal algorithm). $n D R$ is nearly to 0 .

In summary, SM method is more convenient in practical application than GM.

\section{Conclusion}

In this paper, the motion characteristics analysis of PRRPR-S robot is discussed; GM method overcomes the defects of dual redundancy sequence and stochastic of GA. The experiment in Section 3 proved that GA in GM has little effect. SM gets good result directly and more quickly (about half the time consumed in GM).

Even though the kinematic solution method is for PRRPR-S robot, by using the idea of subworkspace and motion path, combining with pattern search, any other type of redundant robot can get the inverse kinematic solution. This is the significance of SM method.

\section{Conflicts of Interest}

The author declares that there are no conflicts of interest related to this paper. 


\section{References}

[1] J. He, S. Wang, and C. Wang, "Application analysis of camera robot in flim vitrual manufacture," Advanced Motion Picture Technolgy, vol. 10, pp. 44-45, 2017.

[2] Z. Lu, Theory and Application of Redundancy Robot, China Machine Press, 2007.

[3] A. Liegeois, "Automatic supervisory control of the configuration and behavior of multibodymechanisms," IEEE Transactions on Systems Man \& Cybernetics, vol. 7, no. 12, pp. 868-871, 1977.

[4] J. K. Parker, A. R. Khoogar, and D. E. Goldberg, "Inverse kinematics of redundant robots using genetic algorithms," in Proceedings of the IEEE International Conference on Robotics and Automation, 1989, vol. 1, pp. 271-276, Scottsdale, Ariz, USA, 2002.

[5] Y. W. Sung, D. K. Cho, and M. J. Chung, "A constrained optimization approach to resolving manipulator redundancy," Journal of Robotic Systems, vol. 13, no. 5, pp. 275-288, 1996.

[6] S. Masayuki, K. Hiromu, W. K. Yoon, K. Kosei, and K. Kazuhiro, "An analytical solution for the inverse kinematics of a redundant 7DoF Manipulator with link offsets," IEEE Transactions on Robotics, vol. 24, no. 5, pp. 1131-1142, 2008.

[7] G. K. Singh and J. Claassens, "An analytical solution for the inverse kinematics of a redundant 7DoF manipulator with link offsets," in Proceedings of the 23rd IEEE/RSJ 2010 International Conference on Intelligent Robots and Systems, IROS 2010, pp. 2976-2982, Taipei, Taiwan, October 2010.

[8] L. Zhang, J. He, and S. Wang, "Inverse Kinematic Solutions of Dual Redundant Camera Robot Based on Genetic Algorithm," Mathematical Problems in Engineering, vol. 2017, 17 pages, 2017. 


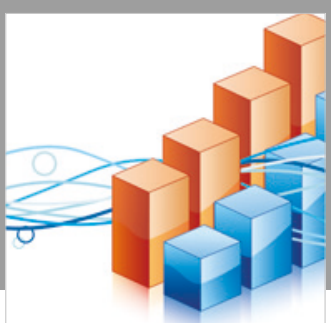

Advances in

Operations Research

\section{-n-m}
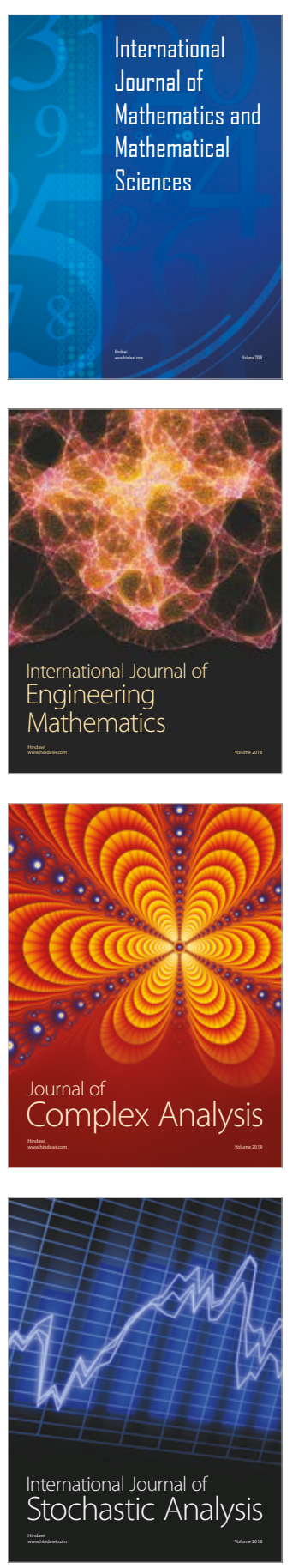
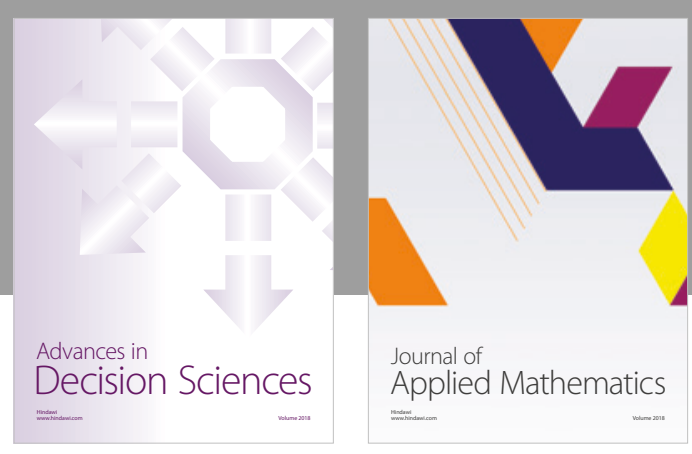

Journal of

Applied Mathematics
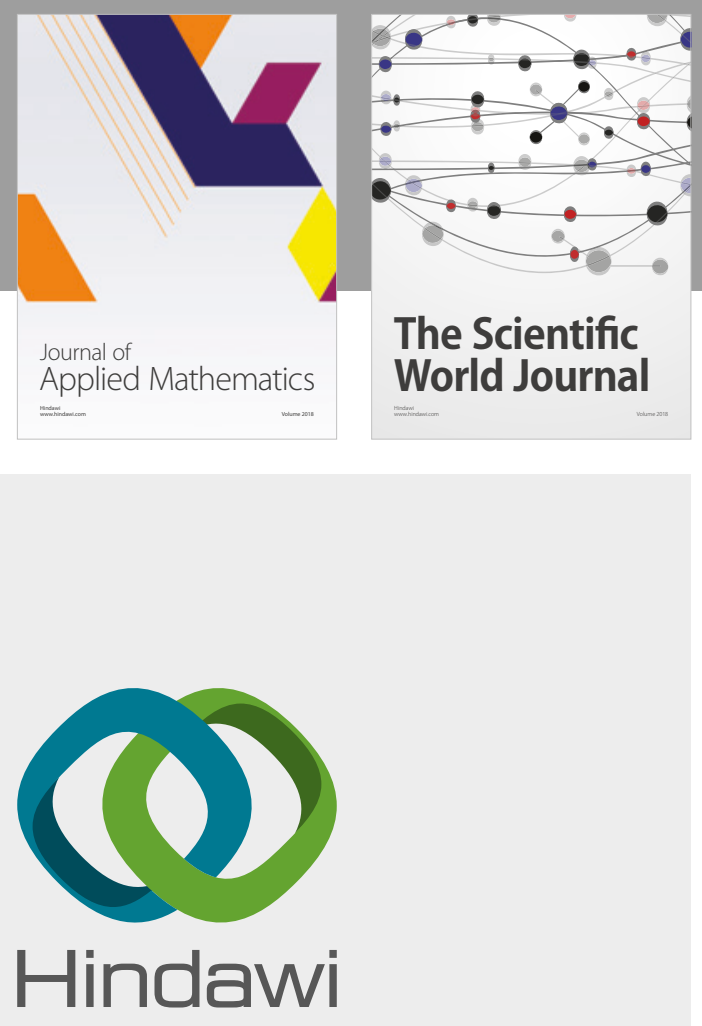

Submit your manuscripts at

www.hindawi.com

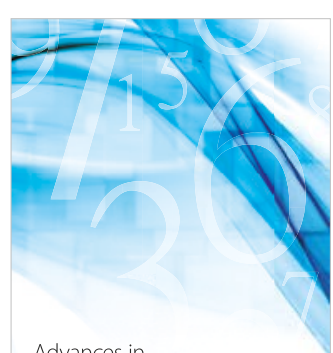

Advances in
Numerical Analysis
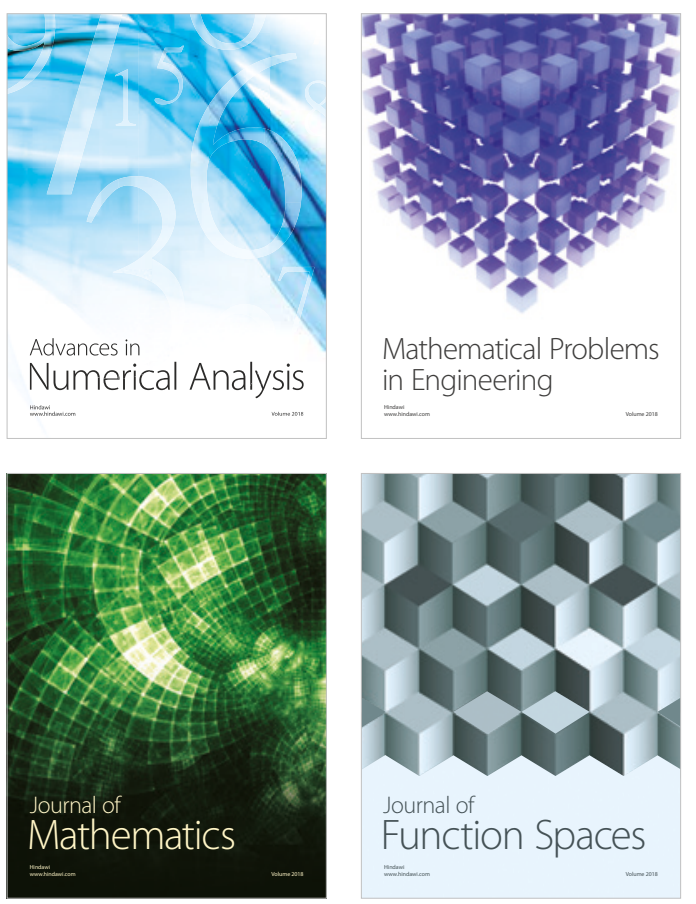

Mathematical Problems in Engineering

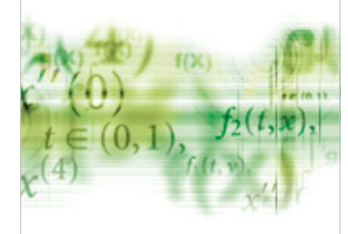

International Journal of

Differential Equations

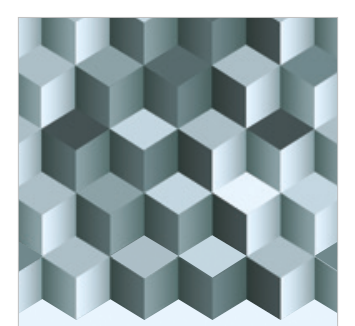

Journal of

Function Spaces

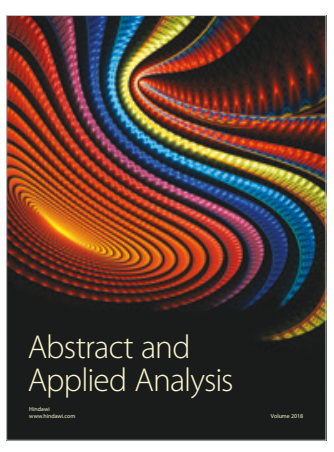

The Scientific

World Journal

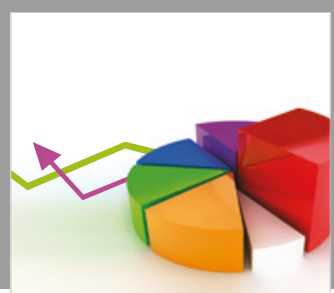

Journal of

Probability and Statistics
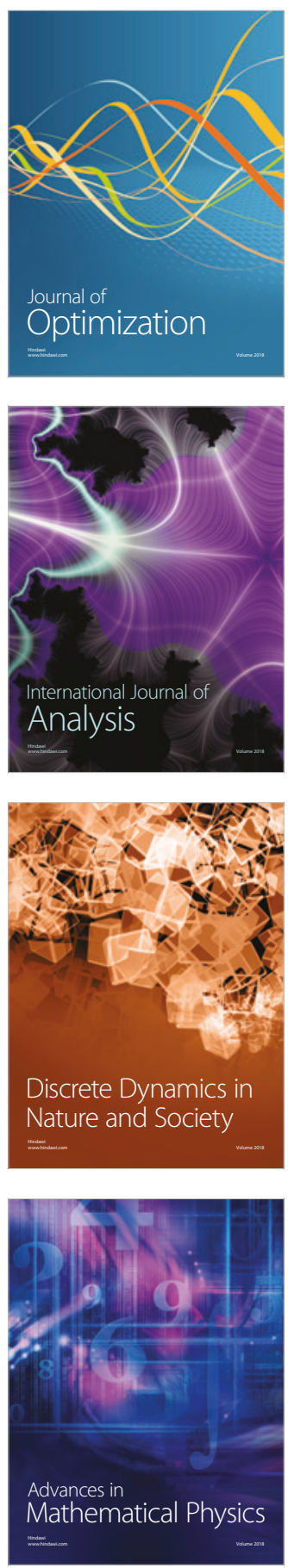